\title{
Relativistic Random-Phase Approximation with density-dependent meson-nucleon couplings
}

\author{
T. Nikšić \\ Physik-Department der Technischen Universität München, D-85748 Garching, Germany and \\ Physics Department, University of Zagreb, 10000 Zagreb, Croatia \\ D. Vretenar \\ Physics Department, University of Zagreb, 10000 Zagreb, Croatia \\ P. Ring \\ Physik-Department der Technischen Universität München, D-85748 Garching, Germany
}

(Dated: October 27, 2018)

\begin{abstract}
The matrix equations of the relativistic random-phase approximation (RRPA) are derived for an effective Lagrangian characterized by density-dependent meson-nucleon vertex functions. The explicit density dependence of the meson-nucleon couplings introduces rearrangement terms in the residual two-body interaction, that are essential for a quantitative description of excited states. Illustrative calculations of the isoscalar monopole, isovector dipole and isoscalar quadrupole response of ${ }^{208} \mathrm{~Pb}$, are performed in the fully self-consistent RRPA framework, based on effective interactions with a phenomenological density dependence adjusted to nuclear matter and ground-state properties of spherical nuclei. The comparison of the RRPA results on multipole giant resonances with experimental data constrains the parameters that characterize the isoscalar and isovector channel of the density-dependent effective interactions.

PACS numbers: 21.60.-n, 21.30.Fe, 21.65.+f, 21.10.-k
\end{abstract}




\section{INTRODUCTION}

The success of models based on the Relativistic Mean Field (RMF) [1] approximation in describing structure phenomena, not only in nuclei along the valley of $\beta$-stability, but also in exotic nuclei with extreme isospin values and close to the particle drip lines, has also renewed the interest in theoretical studies based on the Relativistic Random Phase Approximation (RRPA). Although several RRPA implementations have been available since the eighties, only very recently RRPA-based calculations have reached a level on which a quantitative comparison with experimental data became possible. Two points are essential for the successful application of the RRPA in the description of dynamical properties of finite nuclei: (i) the use of effective Lagrangians with nonlinear self-interaction terms, and (ii) the fully consistent treatment of the Dirac sea of negative energy states. Many studies over the last decade have shown that the inclusion of nonlinear meson terms in meson-exchange RMF models, or nonlinear nucleon self-interaction terms in relativistic point-coupling models, is absolutely necessary in order to reproduce ground-state properties of spherical and deformed nuclei on a quantitative level. Techniques which enable the inclusion of nonlinear meson interaction terms in the RRPA framework, however, have been developed only recently in the calculation of the relativistic linear response [2], and in the solution of the RRPA-matrix equation [3]. For a quantitative description of excited states, the RRPA configuration space must include not only the usual particle-hole states, but also pair-configurations formed from occupied states in the Fermi sea and empty negative-energy states in the Dirac sea. Even though it was known for a long time that the inclusion of configurations built from occupied positive-energy states and empty negative-energy states is essential for current conservation and the decoupling of spurious states [4, only recently it has been shown that the fully consistent inclusion of the Dirac sea of negative energy states in the RRPA is essential for a quantitative comparison with the experimental excitation energies of giant resonances [3, 5].

The RRPA with nonlinear meson interaction terms, and with a configuration space that includes the Dirac sea of negative-energy state, has been very successfully employed in studies of nuclear compressional modes [3, 6, 7], of multipole giant resonances and of lowlying collective states in spherical nuclei [8], of the evolution of the low-lying isovector dipole response in nuclei with a large neutron excess [9, 10], and of toroidal dipole resonances [11].

An interesting alternative to the highly successful RMF models with nonlinear self- 
interaction terms, is an effective hadron field theory with medium dependent meson-nucleon vertices. Such an approach retains the basic structure of the relativistic mean-field framework, but could be more directly related to the underlying microscopic description of nuclear interactions. In particular, the density dependent relativistic hadron field (DDRH) model [12] has been successfully applied in the calculation of nuclear matter and ground-state properties of spherical nuclei [13], and extended to hypernuclei [14], neutron star matter [15], and asymmetric nuclear matter and exotic nuclei [16]. Very recently, in Ref. [17] we have extended the relativistic Hartree-Bogoliubov (RHB) model [18] to include density dependent meson-nucleon couplings. The effective Lagrangian is characterized by a phenomenological density dependence of the $\sigma, \omega$ and $\rho$ meson-nucleon vertex functions, adjusted to properties of nuclear matter and finite nuclei. It has been shown that, in comparison with standard RMF effective interactions with nonlinear meson-exchange terms, the density-dependent meson-nucleon couplings significantly improve the description of symmetric and asymmetric nuclear matter, and of isovector ground-state properties of $N \neq Z$ nuclei. This is, of course, very important for the extension of RMF-based models to exotic nuclei far from $\beta$-stability (description of the neutron skin, the neutron halo, pygmy isovector dipole resonances), and for applications in the field of nuclear astrophysics.

In this work we derive the RRPA with density-dependent meson-nucleon couplings. Just as in the static case the single-nucleon Dirac equation includes the additional rearrangement self-energies that result from the variation of the vertex functionals with respect to the nucleon field operators, the explicit density dependence of the meson-nucleon couplings introduces rearrangement terms in the residual interaction of the RRPA. The rearrangement contribution is essential for a quantitative analysis of excited states in the RRPA framework. In Sec. 【 we present the formalism of the relativistic RPA with density-dependent mesonnucleon couplings, and derive the RRPA equations in the small amplitude limit of the timedependent RMF. The results of an illustrative calculation of multipole giant resonances in ${ }^{208} \mathrm{~Pb}$ are analyzed in Sec. $\mathbb{I I I}$. Section $\mathbb{\mathbb { V }}$ contains the summary and the conclusions. 


\section{FORMALISM OF THE RELATIVISTIC RANDOM-PHASE APPROXIMA- TION WITH DENSITY-DEPENDENT MESON-NUCLEON COUPLINGS}

The standard density dependent relativistic hadron field (DDRH) model [12 for nuclear matter and finite nuclei is defined by the Lagrangian density

$$
\begin{aligned}
\mathcal{L}= & \bar{\psi}(i \boldsymbol{\gamma} \cdot \boldsymbol{\partial}-m) \psi+\frac{1}{2}(\partial \sigma)^{2}-\frac{1}{2} m_{\sigma}^{2} \sigma^{2} \\
& -\frac{1}{4} \Omega_{\mu \nu} \Omega^{\mu \nu}+\frac{1}{2} m_{\omega}^{2} \omega^{2}-\frac{1}{4} \overrightarrow{\mathrm{R}}_{\mu \nu} \overrightarrow{\mathrm{R}}^{\mu \nu}+\frac{1}{2} m_{\rho}^{2} \vec{\rho}^{2}-\frac{1}{4} \mathrm{~F}_{\mu \nu} \mathrm{F}^{\mu \nu} \\
& -g_{\sigma} \bar{\psi} \sigma \psi-g_{\omega} \bar{\psi} \gamma \cdot \omega \psi-g_{\rho} \bar{\psi} \gamma \cdot \vec{\rho} \vec{\tau} \psi-e \bar{\psi} \gamma \cdot A \frac{\left(1-\tau_{3}\right)}{2} \psi
\end{aligned}
$$

Vectors in isospin space are denoted by arrows, and bold-faced symbols indicate vectors in ordinary three-dimensional space. The Dirac spinor $\psi$ denotes the nucleon with mass $m . m_{\sigma}$, $m_{\omega}$, and $m_{\rho}$ are the masses of the $\sigma$-meson, the $\omega$-meson, and the $\rho$-meson. $g_{\sigma}, g_{\omega}$, and $g_{\rho}$ are the corresponding coupling constants for the mesons to the nucleon. $e^{2} / 4 \pi=1 / 137.036$. The coupling constants and the mass of the $\sigma$-meson are treated as free parameters, adjusted to reproduce nuclear matter properties and ground-state properties of finite nuclei. $\Omega^{\mu \nu}, \vec{R}^{\mu \nu}$, and $F^{\mu \nu}$ are the field tensors of the vector fields $\omega, \rho$, and of the photon:

$$
\begin{aligned}
& \Omega^{\mu \nu}=\partial^{\mu} \omega^{\nu}-\partial^{\nu} \omega^{\mu} \\
& \vec{R}^{\mu \nu}=\partial^{\mu} \vec{\rho}^{\nu}-\partial^{\nu} \vec{\rho}^{\mu} \\
& F^{\mu \nu}=\partial^{\mu} A^{\nu}-\partial^{\nu} A^{\mu} .
\end{aligned}
$$

The meson-nucleon couplings $g_{\sigma}, g_{\omega}$, and $g_{\rho}$ are assumed to be vertex functions of Lorentzscalar bilinear forms of the nucleon field operators. In most applications of the densitydependent hadron field theory these couplings are chosen as functions of the vector density $\rho_{v}=\sqrt{j_{\mu} j^{\mu}}$, with $j_{\mu}=\bar{\psi} \gamma_{\mu} \psi$. Alternatively, the couplings could be functionals of the scalar density $\rho_{s}=\bar{\psi} \psi$. It has been shown, however, that the vector density dependence produces better results for finite nuclei [12], and provides a more natural relation between the selfenergies of the density-dependent hadron field theory and the Dirac-Brueckner microscopic self-energies [16]. In the present work we choose the vector density dependence for the meson-nucleon couplings.

The single-nucleon Dirac equation is derived by the variation of the Lagrangian (1) with respect to $\bar{\psi}$

$$
i \partial_{t} \psi_{i}=\left\{\boldsymbol{\alpha}[-i \boldsymbol{\nabla}-\boldsymbol{V}(r, t)]+V(\boldsymbol{r}, t)+\boldsymbol{\beta}(m+S(\boldsymbol{r}, t))+\Sigma_{0}^{R}(\boldsymbol{r}, t)\right\} \psi_{i} .
$$


The Dirac hamiltonian contains the scalar and vector nucleon self-energies defined by the following relations:

$$
\begin{gathered}
S(\boldsymbol{r}, t)=g_{\sigma}\left(\rho_{v}\right) \sigma(\boldsymbol{r}, t) \\
V_{\mu}(\boldsymbol{r}, t)=g_{\omega}\left(\rho_{v}\right) \omega_{\mu}(\boldsymbol{r}, t)+g_{\rho}\left(\rho_{v}\right) \vec{\tau} \cdot \vec{\rho}_{\mu}(\boldsymbol{r}, t)+e \frac{\left(1-\tau_{3}\right)}{2} A_{\mu}(\boldsymbol{r}, t) .
\end{gathered}
$$

The density dependence of the vertex functions $g_{\sigma}, g_{\omega}$, and $g_{\rho}$ produces the rearrangement contribution to the vector self-energy

$$
\Sigma_{0}^{R}(\boldsymbol{r}, t)=\frac{\partial g_{\omega}}{\partial \rho_{v}} \bar{\psi} \gamma^{\nu} \psi \omega_{\nu}+\frac{\partial g_{\rho}}{\partial \rho_{v}} \bar{\psi} \gamma^{\nu} \vec{\tau} \psi \cdot \vec{\rho}_{\nu}+\frac{\partial g_{\sigma}}{\partial \rho_{v}} \bar{\psi} \psi \sigma
$$

The inclusion of the rearrangement self-energies is essential for the energy-momentum conservation and the thermodynamical consistency of the model [12, 13].

In the time-dependent RMF model [19] one usually neglects the retardation effects for the meson fields, and the self-energies are determined at each time by the solutions of the Klein-Gordon and Poisson equations:

$$
\begin{aligned}
{\left[-\Delta+m_{\sigma}^{2}\right] \sigma(\boldsymbol{r}, t) } & =-g_{\sigma}\left(\rho_{v}\right) \rho_{s}(\boldsymbol{r}, t) \\
{\left[-\Delta+m_{\omega}^{2}\right] \omega_{\mu}(\boldsymbol{r}, t) } & =g_{\omega}\left(\rho_{v}\right) j_{\mu}(\boldsymbol{r}, t), \\
{\left[-\Delta+m_{\rho}^{2}\right] \vec{\rho}_{\mu}(\boldsymbol{r}, t) } & =g_{\rho}\left(\rho_{v}\right) \vec{j}_{\mu}(\boldsymbol{r}, t), \\
-\Delta A_{\mu}(\boldsymbol{r}, t) & =e j_{c \mu}(\boldsymbol{r}, t) .
\end{aligned}
$$

This approximation is justified by the large masses in the meson propagators. Retardation effects can be neglected because of the short range of the corresponding meson exchange forces. The explicit solutions of Eqs. (9) read, respectively,

$$
\begin{aligned}
\sigma(\boldsymbol{r}, t) & =-\int g_{\sigma}\left(\rho_{v}\right) D_{\sigma}\left(\boldsymbol{r}, \boldsymbol{r}^{\prime}\right) \rho_{s}\left(\boldsymbol{r}^{\prime}, t\right) d^{3} r^{\prime} \\
\omega_{\mu}(\boldsymbol{r}, t) & =\int g_{\omega}\left(\rho_{v}\right) D_{\omega}\left(\boldsymbol{r}, \boldsymbol{r}^{\prime}\right) j_{\mu}\left(\boldsymbol{r}^{\prime}, t\right) d^{3} r^{\prime}, \\
\vec{\rho}_{\mu}(\boldsymbol{r}, t) & =\int g_{\rho}\left(\rho_{v}\right) D_{\rho}\left(\boldsymbol{r}, \boldsymbol{r}^{\prime}\right) \vec{j}_{\mu}\left(\boldsymbol{r}^{\prime}, t\right) d^{3} r^{\prime}, \\
A_{\mu}(\boldsymbol{r}, t) & =e \int D_{c}\left(\boldsymbol{r}, \boldsymbol{r}^{\prime}\right) j_{c \mu}\left(\boldsymbol{r}^{\prime}, t\right) d^{3} r^{\prime}
\end{aligned}
$$

with the Yukawa propagators

$$
D_{\phi}\left(\boldsymbol{r}, \boldsymbol{r}^{\prime}\right)=\frac{1}{4 \pi} \frac{\mathrm{e}^{-m_{\phi}\left|\boldsymbol{r}-\boldsymbol{r}^{\prime}\right|}}{\left|\boldsymbol{r}-\boldsymbol{r}^{\prime}\right|},
$$

where $\phi$ denotes the $\sigma, \omega$, and $\rho$ mesons, and the photon. 
The sources of the fields are the nucleon densities and currents calculated in the no-sea approximation

$$
\begin{aligned}
\rho_{s}(\boldsymbol{r}, t) & =\sum_{i=1}^{A} \bar{\psi}_{i}(\boldsymbol{r}, t) \psi_{i}(\boldsymbol{r}, t), \\
j_{\mu}(\boldsymbol{r}, t) & =\sum_{i=1}^{A} \bar{\psi}_{i}(\boldsymbol{r}, t) \gamma_{\mu} \psi_{i}(\boldsymbol{r}, t), \\
\vec{j}_{\mu}(\boldsymbol{r}, t) & =\sum_{i=1}^{A} \bar{\psi}_{i}(\boldsymbol{r}, t) \vec{\tau} \gamma_{\mu} \psi_{i}(\boldsymbol{r}, t), \\
j_{c \mu}(\boldsymbol{r}, t) & =\sum_{i=1}^{Z} \bar{\psi}_{i}(\boldsymbol{r}, t) \gamma_{\mu} \psi_{i}(\boldsymbol{r}, t) .
\end{aligned}
$$

where the summation is over all A occupied states in the Fermi sea, i.e. only occupied single-nucleon states with positive energy explicitly contribute to the nucleon self-energies. Even though the stationary solutions for the negative-energy states do not contribute to the densities in the no-sea approximation, their contribution is implicitly included in the time-evolution of the nuclear system [0. [19].

The Relativistic Random Phase Approximation (RRPA) represents the small amplitude limit of the time-dependent relativistic mean-field theory. In the remainder of this section we will derive the RRPA equations with density-dependent meson-nucleon couplings from the response of the density matrix $\hat{\rho}(t)$ to an external field

$$
\hat{F}(t)=\hat{F} \mathrm{e}^{-i \omega t}+\text { h.c. },
$$

which oscillates with a small amplitude. In the single-particle space this field is represented by the operator

$$
\hat{f}(t)=\sum_{k l} f_{k l}(t) \hat{a}_{k}^{\dagger} \hat{a}_{l} .
$$

The expression for the single-particle density matrix reads

$$
\hat{\rho}\left(\mathbf{r}, \mathbf{r}^{\prime}, t\right)=\sum_{i=1}^{A}\left|\psi_{i}(\mathbf{r}, t)\right\rangle\left\langle\psi_{i}\left(\mathbf{r}^{\prime}, t\right)\right| .
$$

By writing the Dirac spinor in terms of large and small components

$$
\left|\psi_{i}(\mathbf{r}, t)\right\rangle=\left(\begin{array}{r}
f_{i}(\mathbf{r}, t) \\
i g_{i}(\mathbf{r}, t)
\end{array}\right)
$$

the density matrix takes the form

$$
\rho\left(\mathbf{r}, \mathbf{r}^{\prime}, t\right)=\left(\begin{array}{rr}
\sum_{i=1}^{A} f_{i}(\mathbf{r}, t) f_{i}^{\dagger}\left(\mathbf{r}^{\prime}, t\right) & -i \sum_{i=1}^{A} f_{i}(\mathbf{r}, t) g_{i}^{\dagger}\left(\mathbf{r}^{\prime}, t\right) \\
i \sum_{i=1}^{A} g_{i}(\mathbf{r}, t) f_{i}^{\dagger}\left(\mathbf{r}^{\prime}, t\right) & \sum_{i=1}^{A} g_{i}(\mathbf{r}, t) g_{i}^{\dagger}\left(\mathbf{r}^{\prime}, t\right)
\end{array}\right)
$$


The equation of motion for the density operator reads

$$
i \partial_{t} \hat{\rho}=[\hat{h}(\hat{\rho})+\hat{f}(t), \hat{\rho}]
$$

and in the small amplitude limit the density matrix is expanded to linear order

$$
\hat{\rho}(t)=\hat{\rho}^{(0)}+\delta \hat{\rho}(t)
$$

where $\hat{\rho}^{(0)}$ is the stationary ground-state density. From the definition of the density matrix (15), it follows that $\hat{\rho}(t)$ is a projector at all times, i.e. $\hat{\rho}(t)^{2}=\hat{\rho}(t)$. In particular, this means that the eigenvalues of $\hat{\rho}^{(0)}$ are 0 and 1 . In the non-relativistic case particle states above the Fermi level correspond to the eigenvalue 0, and hole states in the Fermi sea correspond to the eigenvalue 1 . In the relativistic case, one also has to take into account states from the Dirac sea. In the no-sea approximation these states are not occupied, i.e. they correspond to the eigenvalue 0 of the density matrix.

$$
\rho_{k l}^{(0)}=\delta_{k l} \rho_{k}^{(0)}=\left\{\begin{array}{l}
0 \text { for unoccupied states above the Fermi level (index } p) \\
1 \text { for occupied states in the Fermi sea (index } h) \\
0 \text { for unoccupied states in the Dirac sea (index } \alpha)
\end{array}\right.
$$

$\hat{\rho}(t)^{2}=\hat{\rho}(t)$ also implies, in leading order,

$$
\hat{\rho}^{(0)} \delta \hat{\rho}+\delta \hat{\rho} \hat{\rho}^{(0)}=\delta \hat{\rho}
$$

and this means that the only non-vanishing matrix elements of $\delta \hat{\rho}$ are: $\delta \rho_{p h}, \delta \rho_{h p}, \delta \rho_{\alpha h}$, and $\delta \rho_{h \alpha}$. These are determined by the solution of equation (18), which in the linear approximation reads

$$
i \partial_{t} \delta \hat{\rho}=\left[\hat{h}^{(0)}, \delta \hat{\rho}\right]+\left[\frac{\partial \hat{h}}{\partial \rho} \delta \rho, \hat{\rho}^{(0)}\right]+\left[\hat{f}, \hat{\rho}^{(0)}\right]
$$

with

$$
\frac{\partial \hat{h}}{\partial \rho} \delta \rho=\sum_{p h} \frac{\partial \hat{h}}{\partial \rho_{p h}} \delta \rho_{p h}+\frac{\partial \hat{h}}{\partial \rho_{h p}} \delta \rho_{h p}+\sum_{\alpha h} \frac{\partial \hat{h}}{\partial \rho_{\alpha h}} \delta \rho_{\alpha h}+\frac{\partial \hat{h}}{\partial \rho_{h \alpha}} \delta \rho_{h \alpha} .
$$

Under the influence of the external field (13), in the small amplitude limit $\delta \rho$ also exhibits the harmonic time dependence $\mathrm{e}^{-i \omega t}$. Taking into account that $\hat{h}_{k l}^{(0)}=\delta_{k l} \epsilon_{k}$ is diagonal in the stationary basis, the resulting RRPA equations read

$$
\left(\omega-\epsilon_{p}+\epsilon_{h}\right) \delta \rho_{p h}=f_{p h}+\sum_{p^{\prime} h^{\prime}} V_{p h^{\prime} h p^{\prime}} \delta \rho_{p^{\prime} h^{\prime}}+V_{p p^{\prime} h h^{\prime}} \delta \rho_{h^{\prime} p^{\prime}}+\sum_{\alpha^{\prime} h^{\prime}} V_{p h^{\prime} h \alpha^{\prime}} \delta \rho_{\alpha^{\prime} h^{\prime}}+V_{p \alpha^{\prime} h h^{\prime}} \delta \rho_{h^{\prime} \alpha^{\prime}}
$$




$$
\begin{aligned}
& \left(\omega-\epsilon_{\alpha}+\epsilon_{h}\right) \delta \rho_{\alpha h}=f_{\alpha h}+\sum_{p^{\prime} h^{\prime}} V_{\alpha h^{\prime} h p^{\prime}} \delta \rho_{p^{\prime} h^{\prime}}+V_{\alpha p^{\prime} h h^{\prime}} \delta \rho_{h^{\prime} p^{\prime}}+\sum_{\alpha^{\prime} h^{\prime}} V_{\alpha h^{\prime} h \alpha^{\prime}} \delta \rho_{\alpha^{\prime} h^{\prime}}+V_{\alpha \alpha^{\prime} h h^{\prime}} \delta \rho_{h^{\prime} \alpha^{\prime}} \\
& \left(\omega-\epsilon_{h}+\epsilon_{p}\right) \delta \rho_{h p}=f_{h p}+\sum_{p^{\prime} h^{\prime}} V_{h h^{\prime} p p^{\prime}} \delta \rho_{p^{\prime} h^{\prime}}+V_{h p^{\prime} p h^{\prime}} \delta \rho_{h^{\prime} p^{\prime}}+\sum_{\alpha^{\prime} h^{\prime}} V_{h h^{\prime} p \alpha^{\prime}} \delta \rho_{\alpha^{\prime} h^{\prime}}+V_{h \alpha^{\prime} p h^{\prime}} \delta \rho_{h^{\prime} \alpha^{\prime}} \\
& \left(\omega-\epsilon_{h}+\epsilon_{\alpha}\right) \delta \rho_{h \alpha}=f_{h \alpha}+\sum_{p^{\prime} h^{\prime}} V_{h h^{\prime} \alpha p^{\prime}} \delta \rho_{p^{\prime} h^{\prime}}+V_{h p^{\prime} \alpha h^{\prime}} \delta \rho_{h^{\prime} p^{\prime}}+\sum_{\alpha^{\prime} h^{\prime}} V_{h h^{\prime} \alpha \alpha^{\prime}} \delta \rho_{\alpha^{\prime} h^{\prime}}+V_{h \alpha^{\prime} \alpha h^{\prime}} \delta \rho_{h^{\prime} \alpha^{\prime}}
\end{aligned}
$$

or, in matrix form

$$
\left[\omega\left(\begin{array}{cc}
1 & 0 \\
0 & -1
\end{array}\right)-\left(\begin{array}{cc}
A & B \\
B^{*} & A^{*}
\end{array}\right)\right]\left(\begin{array}{c}
X \\
Y
\end{array}\right)=\left(\begin{array}{c}
F \\
\bar{F}
\end{array}\right) .
$$

The RRPA matrices $A$ and $B$ read

$$
\begin{aligned}
& A=\left(\begin{array}{cc}
\left(\epsilon_{p}-\epsilon_{h}\right) \delta_{p p^{\prime}} \delta_{h h^{\prime}} & \\
& \left(\epsilon_{\alpha}-\epsilon_{h}\right) \delta_{\alpha \alpha^{\prime}} \delta_{h h^{\prime}}
\end{array}\right)+\left(\begin{array}{cc}
V_{p h^{\prime} h p^{\prime}} & V_{p h^{\prime} h \alpha^{\prime}} \\
V_{\alpha h^{\prime} h p^{\prime}} & V_{\alpha h^{\prime} h \alpha^{\prime}}
\end{array}\right) \\
& B=\left(\begin{array}{cc}
V_{p p^{\prime} h h^{\prime}} & V_{p \alpha^{\prime} h h^{\prime}} \\
V_{\alpha p^{\prime} h h^{\prime}} & V_{\alpha \alpha^{\prime} h h^{\prime}}
\end{array}\right)
\end{aligned}
$$

and the amplitudes $X$ and $Y$ are defined

$$
X=\left(\begin{array}{c}
\delta \rho_{p h} \\
\delta \rho_{\alpha h}
\end{array}\right), \quad Y=\left(\begin{array}{c}
\delta \rho_{h p} \\
\delta \rho_{h \alpha}
\end{array}\right) .
$$

The vectors which represent the external field contain the matrix elements

$$
F=\left(\begin{array}{c}
f_{p h} \\
f_{\alpha h}
\end{array}\right), \quad \bar{F}=\left(\begin{array}{c}
f_{h p} \\
f_{h \alpha}
\end{array}\right) .
$$

In the self-consistent RRPA the matrix elements of the residual interaction are derived from the Dirac hamiltonian of Eq. (5):

$$
\begin{aligned}
V_{a b c d} & =\frac{\partial h_{a c}}{\partial \rho_{d b}} \\
& =\int \Psi_{a}^{+}\left(\boldsymbol{r}_{1}\right) \Psi_{b}^{+}\left(\boldsymbol{r}_{2}\right) V\left(\boldsymbol{r}_{1}, \boldsymbol{r}_{2}\right) \Psi_{c}\left(\boldsymbol{r}_{1}\right) \Psi_{d}\left(\boldsymbol{r}_{2}\right) d^{3} r_{1} d^{3} r_{2} .
\end{aligned}
$$

In order to calculate the contributions of each meson field to $V\left(\boldsymbol{r}_{1}, \boldsymbol{r}_{2}\right)$, we expand the meson-nucleon couplings and their derivatives around the ground-state density $\rho_{v}^{0}$

$$
\begin{aligned}
g_{i}\left(\rho_{v}\right) & =g_{i}\left(\rho_{v}^{0}\right)+\left.\frac{\partial g_{i}}{\partial \rho_{v}}\right|_{0} \delta \rho_{v} \\
\frac{\partial g_{i}}{\partial \rho_{v}} & =\left.\frac{\partial g_{i}}{\partial \rho_{v}}\right|_{0}+\left.\frac{\partial^{2} g_{i}}{\partial \rho_{v}^{2}}\right|_{0} \delta \rho_{v} \quad .
\end{aligned}
$$


If for the meson fields appearing in the scalar and vector nucleon self-energies we use the explicit solutions (10) in terms of the meson propagators and nucleon densities and currents, the individual contributions of the meson fields to $V\left(\boldsymbol{r}_{1}, \boldsymbol{r}_{2}\right)$ are obtained from the particlehole matrix element of the Dirac hamiltonian:

- the contribution of the isoscalar-scalar sigma field

$$
\begin{aligned}
V_{\sigma}\left(\boldsymbol{r}_{1}, \boldsymbol{r}_{2}\right) & =-\beta_{1} \beta_{2} g_{\sigma}\left(\rho_{v}\left(\boldsymbol{r}_{1}\right)\right) g_{\sigma}\left(\rho_{v}\left(\boldsymbol{r}_{2}\right)\right) D_{\sigma}\left(\boldsymbol{r}_{1}, \boldsymbol{r}_{2}\right) \\
& -\left\{\beta_{1} \mathbb{1}_{2} \frac{\partial g_{\sigma}}{\partial \rho_{v}\left(\boldsymbol{r}_{1}\right)}+\mathbb{1}_{1} \mathbb{1}_{2} \frac{\partial^{2} g_{\sigma}}{\partial \rho_{v}^{2}\left(\boldsymbol{r}_{1}\right)} \rho_{s}\left(\boldsymbol{r}_{1}\right)+\mathbb{1}_{1} \beta_{2} \frac{\partial g_{\sigma}}{\partial \rho_{v}\left(\boldsymbol{r}_{1}\right)}\right\} \frac{I_{\sigma}\left(r_{1}\right)}{r_{1}} \delta\left(\boldsymbol{r}_{1}-\boldsymbol{r}_{2}\right) \\
& -\left\{\beta_{1} \mathbb{1}_{2} g_{\sigma}\left(\rho_{v}\left(\boldsymbol{r}_{1}\right)\right) \frac{\partial g_{\sigma}}{\partial \rho_{v}\left(\boldsymbol{r}_{2}\right)} \rho_{s}\left(\boldsymbol{r}_{2}\right)+\mathbb{1}_{1} \beta_{2} \frac{\partial g_{\sigma}}{\partial \rho_{v}\left(\boldsymbol{r}_{1}\right)} \rho_{s}\left(\boldsymbol{r}_{1}\right) g_{\sigma}\left(\rho_{v}\left(\boldsymbol{r}_{2}\right)\right)\right. \\
& \left.+\mathbb{1}_{1} \mathbb{1}_{2} \frac{\partial g_{\sigma}}{\partial \rho_{v}\left(\boldsymbol{r}_{1}\right)} \rho_{s}\left(\boldsymbol{r}_{1}\right) \frac{\partial g_{\sigma}}{\partial \rho_{v}\left(\boldsymbol{r}_{2}\right)} \rho_{s}\left(\boldsymbol{r}_{2}\right)\right\} D_{\sigma}\left(\boldsymbol{r}_{1}, \boldsymbol{r}_{2}\right)
\end{aligned}
$$

where

$$
I_{\sigma}\left(r_{1}\right)=\int r g_{\sigma}\left(\rho_{v}(r)\right) D_{\sigma}^{0}\left(r_{1}, r\right) \rho_{s}(r) d r
$$

- the contribution of the isoscalar-vector omega field

$$
\begin{aligned}
V_{\omega}\left(\boldsymbol{r}_{1}, \boldsymbol{r}_{2}\right) & =\left(\beta \gamma^{\mu}\right)_{1}\left(\beta \gamma_{\mu}\right)_{2} g_{\omega}\left(\rho_{v}\left(\boldsymbol{r}_{1}\right)\right) g_{\omega}\left(\rho_{v}\left(\boldsymbol{r}_{2}\right)\right) D_{\omega}\left(\boldsymbol{r}_{1}, \boldsymbol{r}_{2}\right) \\
& +\left\{2 \frac{\partial g_{\omega}}{\partial \rho_{v}\left(\boldsymbol{r}_{1}\right)}+\frac{\partial^{2} g_{\omega}}{\partial \rho_{v}^{2}\left(\boldsymbol{r}_{1}\right)} \rho_{v}\left(\boldsymbol{r}_{1}\right)\right\} \mathbb{1}_{1} \mathbb{1}_{2} \frac{I_{\omega}\left(r_{1}\right)}{r_{1}} \delta\left(\boldsymbol{r}_{1}-\boldsymbol{r}_{2}\right) \\
& +\left\{g_{\omega}\left(\rho_{v}\left(\boldsymbol{r}_{1}\right)\right) \frac{\partial g_{\omega}}{\partial \rho_{v}\left(\boldsymbol{r}_{2}\right)} \rho_{v}\left(\boldsymbol{r}_{2}\right)+\frac{\partial g_{\omega}}{\partial \rho_{v}\left(\boldsymbol{r}_{1}\right)} \rho_{v}\left(\boldsymbol{r}_{1}\right) g_{\omega}\left(\rho_{v}\left(\boldsymbol{r}_{2}\right)\right)\right. \\
& \left.+\frac{\partial g_{\omega}}{\partial \rho_{v}\left(\boldsymbol{r}_{1}\right)} \rho_{v}\left(\boldsymbol{r}_{1}\right) \frac{\partial g_{\omega}}{\partial \rho_{v}\left(\boldsymbol{r}_{2}\right)} \rho_{v}\left(\boldsymbol{r}_{2}\right)\right\} \mathbb{1}_{1} \mathbb{1}_{2} D_{\omega}\left(\boldsymbol{r}_{1}, \boldsymbol{r}_{2}\right),
\end{aligned}
$$

where

$$
I_{\omega}\left(r_{1}\right)=\int r g_{\omega}\left(\rho_{v}(r)\right) D_{\omega}^{0}\left(r_{1}, r\right) \rho_{v}(r) d r
$$

- the contribution of the isovector-vector rho field

$$
\begin{aligned}
V_{\rho}^{1}\left(\boldsymbol{r}_{1}, \boldsymbol{r}_{2}\right) & =\left(\beta \gamma^{\mu}\right)_{1}\left(\beta \gamma_{\mu}\right)_{2} \tau_{1}^{3} \tau_{2}^{3} g_{\rho}\left(\rho_{v}\left(\boldsymbol{r}_{1}\right)\right) g_{\rho}\left(\rho_{v}\left(\boldsymbol{r}_{2}\right)\right) D_{\rho}\left(\boldsymbol{r}_{1}, \boldsymbol{r}_{2}\right) \\
& +\left\{\frac{\partial g_{\rho}}{\partial \rho_{v}\left(\boldsymbol{r}_{1}\right)} \tau_{1}^{3}+\frac{\partial g_{\rho}}{\partial \rho_{v}\left(\boldsymbol{r}_{1}\right)} \tau_{2}^{3}+\frac{\partial^{2} g_{\rho}}{\partial \rho_{v}^{2}\left(\boldsymbol{r}_{1}\right)} \rho_{t v}\left(\boldsymbol{r}_{1}\right)\right\} \mathbb{1}_{1} \mathbb{1}_{2} \frac{I_{\rho}\left(r_{1}\right)}{r_{1}} \delta\left(\boldsymbol{r}_{1}-\boldsymbol{r}_{2}\right) \\
& +\left\{g_{\rho}\left(\rho_{v}\left(\boldsymbol{r}_{1}\right)\right) \tau_{1}^{3} \frac{\partial g_{\rho}}{\partial \rho_{v}\left(\boldsymbol{r}_{2}\right)} \rho_{t v}\left(\boldsymbol{r}_{2}\right)+\frac{\partial g_{\rho}}{\partial \rho_{v}\left(\boldsymbol{r}_{1}\right)} \rho_{t v}\left(\boldsymbol{r}_{1}\right) g_{\rho}\left(\rho_{v}\left(\boldsymbol{r}_{2}\right)\right) \tau_{2}^{3}\right. \\
& \left.+\frac{\partial g_{\rho}}{\partial \rho_{v}\left(\boldsymbol{r}_{1}\right)} \rho_{t v}\left(\boldsymbol{r}_{1}\right) \frac{\partial g_{\rho}}{\partial \rho_{v}\left(\boldsymbol{r}_{2}\right)} \rho_{t v}\left(\boldsymbol{r}_{2}\right)\right\} \mathbb{1}_{1} \mathbb{1}_{2} D_{\rho}\left(\boldsymbol{r}_{1}, \boldsymbol{r}_{2}\right)
\end{aligned}
$$


where

$$
I_{\rho}\left(r_{1}\right)=\int r g_{\rho}\left(\rho_{v}(r)\right) D_{\rho}^{0}\left(r_{1}, r\right) \rho_{t v}(r) d r
$$

- and finally the contribution of the Coulomb field

$$
V_{c}\left(\boldsymbol{r}_{1}, \boldsymbol{r}_{2}\right)=e^{2}\left(\beta \gamma^{\mu}\right)_{1}\left(\beta \gamma_{\mu}\right)_{2} D_{c}\left(\boldsymbol{r}_{1}, \boldsymbol{r}_{2}\right)
$$

the subscripts 1 and 2 of the Dirac matrices refer to particle 1 and 2 , respectively. $\rho_{v}, \rho_{s}$ and $\rho_{t v}$ denote the vector, scalar, and isovector-vector density, respectively, and the derivatives of the meson-nucleon couplings with respect to the vector density are evaluated at groundstate density $\rho_{v}^{0}$. The radial integrals $I_{\phi}(r)(\phi \equiv \sigma, \omega, \rho)$ contain $D_{\phi}^{0}\left(r, r^{\prime}\right)$, which is the radial factor in the first term of the multipole expansion of the Yukawa propagator (11):

$$
D_{\phi}\left(\boldsymbol{r}, \boldsymbol{r}^{\prime}\right)=\frac{1}{r r^{\prime}} \sum_{L=0}^{\infty} D_{\phi}^{L}\left(r, r^{\prime}\right) \sum_{M=-L}^{L} Y_{L M}(\Omega) Y_{L M}^{*}\left(\Omega^{\prime}\right)
$$

We notice that, in addition to the direct contribution of the meson exchange interactions (first terms in Eqs. (32)-(34)), the explicit density dependence of the meson-nucleon couplings introduces a number of rearrangement terms in the residual two-body interaction $V\left(\boldsymbol{r}_{1}, \boldsymbol{r}_{2}\right)$. These rearrangement terms are essential for fully consistent RRPA calculations. Only when their contribution is included in the matrix elements of the residual interaction, it becomes possible to reproduce reasonably well the excitation energies of giant multipole resonances. Without rearrangement terms, one finds discrepancies of the order of several $\mathrm{MeV}$ between the experimental excitation energies and the RRPA peak energies, calculated with relativistic effective interactions that are adjusted to ground state properties of spherical nuclei. A very similar effect is observed in RRPA calculations based on effective forces with non-linear meson self-interactions, when the contribution of the non-linear terms is not included in the matrix elements of the residual interaction [8].

\section{ILLUSTRATIVE RRPA CALCULATIONS: GIANT RESONANCES}

In this section the RRPA with density-dependent meson-nucleon couplings is applied in illustrative calculations of giant resonances in spherical nuclei. In particular, we analyze the isoscalar monopole, the isovector dipole and the isoscalar quadrupole resonances in ${ }^{208} \mathrm{~Pb}$. We will show which isoscalar and isovector properties of the effective mean-field interactions 
affect the multipole strength distributions, and how the results of RRPA calculations can be used to constrain the effective interaction.

For the density dependence of the meson-nucleon couplings we adopt the functionals used in Refs. [13, 16, 17]. The coupling of the $\sigma$-meson and $\omega$-meson to the nucleon field reads

$$
g_{i}(\rho)=g_{i}\left(\rho_{\text {sat }}\right) f_{i}(x) \quad \text { for } \quad i=\sigma, \omega
$$

where

$$
f_{i}(x)=a_{i} \frac{1+b_{i}\left(x+d_{i}\right)^{2}}{1+c_{i}\left(x+d_{i}\right)^{2}}
$$

is a function of $x=\rho / \rho_{\text {sat }}$, and $\rho_{\text {sat }}$ denotes the baryon density at saturation in symmetric nuclear matter. The eight real parameters in (38) are not independent. The five constraints $f_{i}(1)=1, f_{\sigma}^{\prime \prime}(1)=f_{\omega}^{\prime \prime}(1)$, and $f_{i}^{\prime \prime}(0)=0$, reduce the number of independent parameters to three. Three additional parameters in the isoscalar channel are: $g_{\sigma}\left(\rho_{\text {sat }}\right), g_{\omega}\left(\rho_{\text {sat }}\right)$, and $m_{\sigma}$ - the mass of the phenomenological sigma-meson. For the $\rho$-meson coupling the functional form of the density dependence is suggested by DB calculations of asymmetric nuclear matter 20

$$
g_{\rho}(\rho)=g_{\rho}\left(\rho_{\mathrm{sat}}\right) \exp \left[-a_{\rho}(x-1)\right] .
$$

The isovector channel is parameterized by $g_{\rho}\left(\rho_{\text {sat }}\right)$ and $a_{\rho}$. For the masses of the $\omega$ and $\rho$ mesons usually the free values are used: $m_{\omega}=783 \mathrm{MeV}$ and $m_{\rho}=763 \mathrm{MeV}$. In principle one could also consider the density dependence of the meson masses. However, since the effective meson-nucleon coupling in nuclear matter is determined by the ratio $g / m$, the choice of a phenomenological density dependence of the couplings makes an explicit density dependence of the masses redundant.

The eight independent parameters, seven coupling parameters and the mass of the $\sigma$ meson, are adjusted to reproduce the properties of symmetric and asymmetric nuclear matter, binding energies, charge radii and neutron radii of spherical nuclei. In particular, in Ref. [17] we have introduced the density-dependent meson-exchange effective interaction

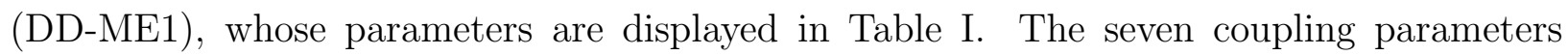
and the $\sigma$-meson mass have been simultaneously adjusted to properties of symmetric and asymmetric nuclear matter, and to ground-state properties (binding energies, charge radii and differences between neutron and proton radii) of twelve spherical nuclei. For the open shell nuclei pairing correlations have been treated in the BCS approximation with empirical pairing gaps (five-point formula). 
In Ref. [17 the relativistic Hartree-Bogoliubov (RHB) model with the density-dependent interaction DD-ME1 in the $p h$-channel, and with the finite range Gogny interaction D1S in the $p p$-channel, has been tested in the analysis of the equations of state for symmetric and asymmetric nuclear matter, and of ground-state properties of the $\mathrm{Sn}$ and $\mathrm{Pb}$ isotopic chains. It has been shown that, as compared to standard non-linear relativistic mean-field effective forces, the interaction DD-ME1 has better isovector properties and therefore provides an improved description of asymmetric nuclear matter, neutron matter and nuclei far from stability.

In the present analysis we perform fully consistent RRPA calculations of isoscalar monopole, isovector dipole and isoscalar quadrupole giant resonances in ${ }^{208} \mathrm{~Pb}$. The singleparticle basis and the particle-hole couplings are obtained from the same effective Lagrangian, and the configuration space includes both particle-hole pairs, as well as pairs formed from hole states and negative-energy states from the Dirac sea. Our starting point is the DD-ME1 effective force, both in the Dirac hamiltonian (5), as well as the residual interaction. We then proceed to construct families of density-dependent interactions with some given characteristic (compressibility, asymmetry energy, etc.), and study the resulting properties of giant resonances.

The isoscalar giant monopole resonance (ISGMR) represents the most simple mode of collective excitations in nuclei. In particular, the ISGMR in heavy nuclei is the only source of experimental information on the nuclear matter compression modulus $K_{\infty}$. This quantity determines basic properties of nuclei, supernovae explosions, neutron stars and heavy-ion collisions. The range of values of $K_{\infty}$ has been deduced from the measured excitation energies of the ISGMR in spherical nuclei. The presently available experimental data set, however, does not limit the range of $K_{\infty}$ to better than $200-300 \mathrm{MeV}$. The microscopic determination of the nuclear matter compressibility is based on the construction of sets of effective interactions that differ mostly by their prediction of the excitation energies of ISGMR, i.e. by the value of $K_{\infty}$, but otherwise reproduce reasonably well experimental data on ground-state nuclear properties [21, 22]. Effective interactions with different values of $K_{\infty}$ are used to calculate bulk ground-state properties of heavy spherical nuclei in a self-consistent mean-field framework, and RPA or time-dependent mean-field calculations are performed for the isoscalar monopole excitations. Such a fully consistent calculation of both ground-state properties, as well as ISGMR excitation energies, restricts the range of possible values for 
$K_{\infty}$. However, since there are also other effects beyond the mean-field level which influence the isoscalar monopole resonance (anharmonicities, pairing, coupling between single-nucleon and collective motion), it has been argued [22] that, rather than on the systematics over the whole periodic table, the determination of the nuclear compressibility should rely more on a good measurement and microscopic calculations of GMR in a single heavy nucleus such as ${ }^{208} \mathrm{~Pb}$. Microscopic calculations have been performed both in the non-relativistic and in the relativistic mean-field framework. Modern non-relativistic Hartree-Fock plus RPA calculations, using both Skyrme and Gogny effective interactions, indicate that the value of $K_{\infty}$ should be in the range $210-220 \mathrm{MeV}$ [21, 22]. In particular, in Ref. 22] a set of effective Gogny forces was generated, which on one hand allow a good description of static properties of nuclei, and on the other hand span the range $210 \leq K_{\infty} \leq 300 \mathrm{MeV}$. It was shown that the RPA calculations reproduce the available experimental data on ISGMR in medium-heavy and heavy nuclei only for $K_{\infty}$ in the range 210-220 MeV. In Ref. [23] it has been shown that even generalized Skyrme forces, with both density- and momentum-dependent terms, can only reproduce the measured breathing mode energies for values of $K_{\infty}$ in the range $215 \pm 15 \mathrm{MeV}$. In relativistic mean-field models based on non-linear meson self-interactions on the other hand, results of both RRPA and time-dependent calculations suggest that empirical GMR energies are best reproduced by an effective force with $K_{\infty} \approx 250-270$ $\mathrm{MeV}$ [6, 24]. It has to be emphasized, however, that even though relativistic calculations have been performed using non-linear effective interactions with different values of $K_{\infty}$, these forces were not constructed specifically with the purpose of determining $K_{\infty}$. Rather, standard non-linear effective interactions have been used, which also exhibit other differences that could affect the microscopic determination of the nuclear matter compressibility.

Starting from DD-ME1, in this work we have generated a consistent set of relativistic density-dependent effective interactions with $220 \leq K_{\infty} \leq 280 \mathrm{MeV}$. The same functional form for the density dependence for the meson-nucleon couplings has been used for these forces and, except for the value of $K_{\infty}$, their parameters have been adjusted to the same set of experimental data on ground-state properties of twelve spherical nuclei [17]. The results of fully consistent RRPA calculations with these forces are shown in Fig. [1, where we display the calculated excitation energies of ISGMR in ${ }^{208} \mathrm{~Pb}$ as function of the nuclear matter compressibility. The shaded region denotes the range of presently available experimental data [25. We notice that, in accordance with the results obtained with relativistic effective 
forces with non-linear meson self-interactions, only the density-dependent interactions with $K_{\infty} \approx 260-270 \mathrm{MeV}$ reproduce the experimental value. We have also verified that ISGMR excitation energies for lighter nuclei, calculated with these particular interactions, are closest to the empirical curve $E_{x} \approx 80 A^{-1 / 3} \mathrm{MeV}$ and that they reproduce the experimental excitation energies. For the density-dependent effective interaction with $K_{\infty}=270 \mathrm{MeV}$, in Fig. \& we display the isoscalar monopole strength distribution and transition densities in ${ }^{208} \mathrm{~Pb}$. The position of the ISGMR peak is at $E=14.1 \mathrm{MeV}$, and we plot the proton, neutron and total isoscalar transition densities.

The present analysis, therefore, confirms that there is a pronounced difference between the values of the nuclear matter compression modulus predicted by microscopic non-relativistic $\left(K_{\infty} \approx 210-230 \mathrm{MeV}\right)$ and relativistic $\left(K_{\infty} \approx 250-270 \mathrm{MeV}\right)$ mean-field plus random phase approximation calculations. The origin of this discrepancy is at present not understood, even though there are some indications that it might be due, at least in part, to the differences in the density dependence of the asymmetry energy predicted by non-relativistic and relativistic models [26].

The calculated properties of isovector dipole giant resonances (IVGDR) will be predominantly determined by the isovector channel of the effective interaction. In particular, the excitation energies of IVGDR can be directly related to the nuclear matter asymmetry energy. The energy per particle of asymmetric nuclear matter can be expanded about the equilibrium density $\rho_{\text {sat }}$ in a Taylor series in $\rho$ and $\alpha$ [27]

$$
E(\rho, \alpha)=E(\rho, 0)+S_{2}(\rho) \alpha^{2}+S_{4}(\rho) \alpha^{4}+\cdots
$$

where

$$
\begin{gathered}
\alpha \equiv \frac{N-Z}{N+Z} . \\
E(\rho, 0)=-a_{v}+\frac{K_{0}}{18 \rho_{\text {sat }}^{2}}\left(\rho-\rho_{\text {sat }}\right)^{2}+\ldots
\end{gathered}
$$

and

$$
S_{2}(\rho)=a_{4}+\frac{p_{0}}{\rho_{\text {sat }}^{2}}\left(\rho-\rho_{\text {sat }}\right)+\frac{\Delta K_{0}}{18 \rho_{\text {sat }}^{2}}\left(\rho-\rho_{\text {sat }}\right)^{2}+\cdots
$$

The empirical value of the asymmetry energy at saturation density (volume asymmetry) $S_{2}\left(\rho_{\text {sat }}\right)=a_{4}=30 \pm 4 \mathrm{MeV}$. The parameter $p_{0}$ defines the linear density dependence of the asymmetry energy, and $\Delta K_{0}$ is the correction to the incompressibility. The contribution of 
the term $S_{4}(\rho) \alpha^{4}$ in (40) is very small in ordinary nuclei and the coefficient is not constrained in the mean-field approximation.

A ground-state nuclear property which is directly determined by the asymmetry energy is the difference between the neutron and the proton radii. In a recent study of neutron radii in non-relativistic and covariant mean-field models [28], the linear correlation between the neutron skin and the symmetry energy has been analyzed. In particular, the analysis has shown that there is a very strong linear correlation between the neutron skin thickness in ${ }^{208} \mathrm{~Pb}$ and the individual parameters that determine the symmetry energy $S_{2}(\rho): a_{4}, p_{0}$ and $\Delta K_{0}$. The empirical value of $r_{n}-r_{p}$ in ${ }^{208} \mathrm{~Pb}(0.20 \pm 0.04 \mathrm{fm}$ from proton scattering data [29], and $0.19 \pm 0.09 \mathrm{fm}$ from the alpha scattering excitation of the isovector giant dipole resonance [30]) places the following constraints on the values of the parameters of the symmetry energy: $a_{4} \approx 30-34 \mathrm{MeV}, 2 \mathrm{Mev} / \mathrm{fm}^{3} \leq p_{0} \leq 4 \mathrm{Mev} / \mathrm{fm}^{3}$, and $-200 \mathrm{MeV}$ $\leq \Delta K_{0} \leq-50 \mathrm{MeV}$

Properties of isovector collective modes in finite nuclei should, in principle, provide additional constraints on the isovector channel of the effective interaction. In an analysis of Skyrme forces and giant resonances in exotic nuclei [31], Reinhard noticed a somewhat surprising property of the IVGDR: while it is true that the excitation energy of this resonance is sensitive to the volume asymmetry $a_{4}$, the resonance energy decreases by increasing the asymmetry energy at saturation. This was qualitatively explained by noticing that an increase in the volume asymmetry is always accompanied by an increase of the slope $p_{0}$, i.e. of the linear density dependence of the asymmetry energy. In order to study this effect in a more quantitative way, we have generated, starting from DD-ME1, a set of eight density dependent effective interactions with $30 \mathrm{MeV} \leq a_{4} \leq 37 \mathrm{MeV}$. The parameters of the density-dependent meson-nucleon couplings have been adjusted in such a way that, while increasing $a_{4}$ in units of $1 \mathrm{MeV}$, the resulting effective interactions still reproduce the same set of data on ground-state properties of spherical nuclei, that was used for the original interaction DD-ME1 [17]. This means that these effective interactions essentially differ only in their description of the asymmetry energy curve as function of the baryon density.

The resulting nuclear matter asymmetry energy curves, and the calculated IVGDR excitation energies in ${ }^{208} \mathrm{~Pb}$, are displayed in Fig. 3. In the upper left panel we plot the RRPA excitation energy of the IVGDR in ${ }^{208} \mathrm{~Pb}$ as function of the volume asymmetry $a_{4}$. Similar to what has been observed in Ref. [31], the resonance energy decreases with increasing $a_{4}$. The 
reason for this decrease is shown in the lower left panel, where we plot the corresponding values of the slope parameter $p_{0}$, which defines the linear density dependence of the asymmetry energy. We notice that, in order to reproduce the bulk properties of spherical nuclei, an increase of $a_{4}$ necessitates a non-linear increase of $p_{0}$. The resulting asymmetry energy curves as functions of the baryon density are shown in the right panel of Fig. 3. The increase of $p_{0}$ with $a_{4}$ implies a transition from a parabolic to an almost linear density dependence of $S_{2}$ in the density region $\rho \leq 0.2 \mathrm{fm}^{-3}$. This means, in particular, that the increase of the asymmetry energy at saturation point will produce an effective decrease of $S_{2}$ below $\rho \approx 0.1 \mathrm{fm}^{-3}$. But this is, of course, the density region characteristic for the IVGDR. We find, therefore, that the excitation energy of the IVGDR decreases with increasing $S_{2}\left(\rho_{\text {sat }}\right) \equiv a_{4}$, because this increase implies a decrease of $S_{2}$ at low densities characteristic for the surface modes. In the upper left panel of Fig. 3 we also compare the calculated IVGDR peak energies for ${ }^{208} \mathrm{~Pb}$, with the experimental value of $13.3 \pm 0.1$ [32]. It appears that the experimental IVGDR excitation energy constrains the nuclear matter asymmetry energy at saturation density to the interval $34 \mathrm{MeV} \leq a_{4} \leq 36 \mathrm{MeV}$. For the effective interaction with $a_{4}=35 \mathrm{MeV}$, in Fig. 1 we display the RRPA isovector dipole strength distribution and the corresponding proton, neutron, and total isovector transition densities for the peak at $13.3 \mathrm{MeV}$ in ${ }^{208} \mathrm{~Pb}$.

Fig. fillustrates what happens when the increase of the nuclear matter asymmetry energy at saturation density is not accompanied by an increase of the slope parameter $p_{0}$. Starting with DD-ME1, which has $a_{4}=33.1 \mathrm{MeV}$, we have generated a set of effective interactions with different values of $a_{4}$, but now they all have the same slope parameter $p_{0}$ (lower left panel), i.e. the parameters are not readjusted to reproduce the data set of ground state properties of spherical nuclei. Binding energies and radii are only approximately reproduced with these effective interactions. The resulting asymmetry energy curves as functions of the baryon density are plotted in the right panel. Since $p_{0}$ is constant, by increasing $a_{4}$ the asymmetry energy $S_{2}$ increases for all densities. As a result, the IVGDR peak energies of ${ }^{208} \mathrm{~Pb}$ increase linearly with $a_{4}$ (upper left panel).

In non-relativistic RPA calculations, the excitation energy of the isoscalar giant quadrupole resonance (ISGQR) can be directly related to the nucleon effective mass that characterizes a given effective interaction. In the non-relativistic mean-field approximation, the total effective mass $m^{*}$ of a nucleon in a nucleus characterizes the energy dependence of an effective local potential that is equivalent to the, generally nonlocal and frequency 
dependent, microscopic nuclear potential [33]. $m^{*}$ is a measure of the density of singlenucleon states around the Fermi surface and, therefore, it affects the giant resonances. For Skyrme interactions, in particular, a linear dependence on $m^{*}$ is found for the RPA excitation energies of the ISGQR. The larger the effective mass, i.e. the higher the density of states around the Fermi surface, the lower is the calculated ISGQR excitation energy. Both the calculation of ground-state properties in spherical nuclei, as well as the RPA results for ISGQR excitation energies, place the following constraint on the nucleon effective mass for Skyrme-type interactions: $m^{*} / m=0.8 \pm 0.1$ [31.

In the relativistic framework the concept of effective nucleon mass is more complicated. In addition to the $k$-mass (characterizes the momentum dependence of the mass operator) and $E$ - mass (characterizes the explicit energy dependence of the mass operator), a third effective mass, the "Lorentz mass" appears in the relativistic approach. It results from different Lorentz transformation properties of the scalar and vector potentials [34, 35]. However, what is usually termed "the relativistic effective mass" that characterizes an effective interaction, is a fourth mass: the Dirac mass [34]

$$
m_{D}=m+S(\boldsymbol{r})
$$

where $m$ is the nucleon mass and $S(\boldsymbol{r})$ is the scalar nucleon self-energy. It has to be emphasized that the Dirac mass should not be identified with the effective mass determined from non-relativistic shell and optical model analyses of experimental data, i.e. with the "non-relativistic-type effective mass". The Dirac mass is determined, on one hand by the binding energy at saturation density in nuclear matter (the effective single-nucleon potential is the sum of the attractive scalar and repulsive vector nucleon self-energies), and on the other hand by the empirical spin-orbit splittings in finite nuclei (the effective single-nucleon spin-orbit potential is proportional to the difference between the scalar and vector self energies). This is the reason why, for virtually all mean-field relativistic effective interactions, $0.55 m \leq m_{D} \leq 0.60 m$.

In contrast to the non-relativistic effective mass, the Dirac mass cannot be related to the ISGQR. On the other hand, we would like to use the isoscalar quadrupole response to constrain the isoscalar properties of our density-dependent effective interactions. This can be done in the following way. We first notice that a particular ratio of isoscalar parameters $b_{i}$ and $c_{i}(i \equiv \sigma, \omega)$ in Eq. (38), characterizes the density of single-nucleon states around the 
Fermi surface. In Fig. 6 we display the average energy gaps between the last occupied and the first unoccupied major shells $<E_{\text {particles }}>-<E_{\text {holes }}>$ in ${ }^{208} \mathrm{~Pb}$, where

$$
<E>=\frac{\sum_{n l j}(2 j+1) E_{n l j}}{\sum_{n l j}(2 j+1)},
$$

and the sums run over occupied (unoccupied) states within a major shell. The average gaps for proton and neutron states are plotted as functions of the parameter $\delta$

$$
\delta=\frac{b_{\sigma} / c_{\sigma}}{b_{\omega} / c_{\omega}}
$$

see Eq. (38). Starting from DD-ME1, we have generated a set of five effective interactions with $0.93 \leq \delta \leq 1.01$. For each interaction the remaining parameters were readjusted to reproduce our standard set of ground-state data for twelve spherical nuclei, as well as the nuclear matter equation of state. The average gap between the last occupied and first unoccupied major shells, both for proton and neutron states, is approximately linearly proportional to $\delta$. This parameter, therefore, plays the role of the inverse of the effective mass. As functions of $\delta$, in Fig. 17 we plot the corresponding centroid energy of the isoscalar quadrupole Hartree response in ${ }^{208} \mathrm{~Pb}$ (upper panel), and the peak energies of the ISGQR obtained by the full RRPA calculation with the five density-dependent interactions (lower panel). The calculated ISGQR excitation energies are compared with the experimental value of $10.9 \pm 0.3 \mathrm{MeV}$ [36] (shaded area). Both the centroids of the Hartree response and the ISGQR peak energies are linearly proportional to $\delta$ and the comparison with experimental data on ISGQR, therefore, places an additional constraint on the parameters that characterize the isoscalar channel of the effective interaction. For $\delta=0.93$, in Fig. \& we plot the RRPA isoscalar quadrupole strength distribution in ${ }^{208} \mathrm{~Pb}$ (left panel), and for the ISGQR peak at $11.2 \mathrm{MeV}$ the proton, neutron, and total isoscalar transition densities. The position of the calculated peak should be compared with the empirical excitation energy $10.9 \pm 0.3$ $\mathrm{MeV}$, and also the $0 \hbar \omega$ low-lying discrete $2^{+}$state at $4.62 \mathrm{MeV}$ is found in good agreement with the experimental value of $4.07 \mathrm{MeV}$.

\section{SUMMARY AND CONCLUSIONS}

During the last decade the standard RMF models with nonlinear meson-exchange effective interactions have been very successfully applied in the description of a variety of nuclear 
structure phenomena. In the last couple of years also the relativistic random-phase approximation (RRPA), based on effective Lagrangians with nonlinear meson self-interaction terms, has been used to investigate properties of low-lying collective states and of giant resonances. The use of nonlinear effective interactions, however, presents not only a number of technical problems, but also the predictive power of models based on these type of interactions appears to be somewhat limited, especially for isovector properties of exotic nuclei far from $\beta$-stability. An interesting alternative are models with density-dependent meson-nucleon vertex functions. Even though these two classes of models are essentially based on the same microscopic structure, i.e. on density dependent interactions, the latter can be more directly related to the underlying microscopic nuclear interactions. In a number of recent analyses it has been also shown that relativistic effective interactions, with explicit density dependence of the meson-nucleon couplings, provide an improved description of asymmetric nuclear matter, neutron matter and nuclei far from stability.

Among the new structure phenomena observed or predicted in nuclei far from stability, one of the most interesting is the evolution of the isovector dipole response in nuclei with a large neutron excess. The multipole response of nuclei with large neutron excess has been the subject of many theoretical studies in recent years, and some predictions have been confirmed by very recent experimental data on low-lying electric dipole strength in neutron rich nuclei [37, 38]. There are, however, many unknowns and this topic presents an interesting challenge for modern theoretical advances. It is, therefore, important to develop also a relativistic framework, based on effective Lagrangians with density-dependent meson-nucleon couplings, in which the dynamics of exotic collective modes in nuclei far from stability can be investigated.

In this work we have derived the RRPA matrix equations in the small amplitude limit of the time-dependent relativistic mean-field theory. The explicit density dependence of the meson-nucleon vertices introduces a number of rearrangement terms in the residual two-body interaction. We have found that the rearrangement contribution to the matrix elements of the RRPA equations is crucial for a quantitative comparison with experimental data on giant resonances. In the present analysis we have performed illustrative RRPA calculations of the isoscalar monopole, isovector dipole and isoscalar quadrupole response of ${ }^{208} \mathrm{~Pb}$. The calculations are fully self-consistent: the single-particle basis and the particle-hole couplings are generated from the same effective Lagrangian, and the RRPA configuration space in- 
cludes both the positive-energy particle-hole pairs, as well as pairs formed from hole states and negative-energy states in the Dirac sea. On one hand, we have tested our approach by comparing the RRPA results for giant resonances with well known experimental data. On the other hand we have also analyzed how the RRPA results on multipole giant resonances can be used to constrain the parameters that characterize the isoscalar and isovector channel of the density-dependent effective Lagrangians. Starting with the recently introduced effective interaction DD-ME1 [17], RRPA calculations have been performed for families of density-dependent interactions with a given characteristic (nuclear matter incompressibility, asymmetry energy etc.).

The analysis of the isoscalar monopole response has shown that only the densitydependent interactions with the nuclear matter compression modulus in the range $K_{\infty} \approx$ $260-270 \mathrm{MeV}$, reproduce the experimental excitation energy of the isoscalar giant monopole resonance in ${ }^{208} \mathrm{~Pb}$. This confirms our previous results obtained with relativistic effective forces with non-linear meson self-interactions and points, once again, to the pronounced difference between the values of the nuclear matter compression modulus predicted by microscopic non-relativistic and relativistic mean-field plus RPA calculations. The RRPA results for the isovector dipole response constrain the isovector channel of the effective interactions. By using interactions with different values of the volume asymmetry energy $a_{4}$, but which otherwise reproduce the same data set of ground-state properties of spherical nuclei, we have shown that the calculated IVGDR peak energy actually decreases by increasing the asymmetry energy at saturation. The comparison with the experimental IVGDR excitation energy constrains the volume asymmetry to the interval $34 \mathrm{MeV} \leq a_{4} \leq 36 \mathrm{MeV}$. In the non-relativistic framework the isoscalar quadrupole response can be related to the effective mass of the mean-field interaction. The concept of effective mass in the relativistic meanfield models is more complicated, and the quantity which is usually termed as "effective mass" cannot be identified with the effective mass determined from non-relativistic shell and optical model analyses of experimental data. Nevertheless, we have shown that a comparison of RRPA results with the empirical ISGQR and with the low-lying $0 \hbar \omega 2^{+}$state, places an additional constraint on the parameters which characterize the isoscalar channel of the density-dependent effective interactions.

The RRPA with density-dependent meson-nucleon couplings presents an important step in the relativistic description of the nuclear many-body problem. In the present work we did 
not attempt an analysis of the multipole response in exotic nuclei far from $\beta$-stability. In order to do that, pairing correlations must be included in the RRPA framework. Work is in progress on the fully self-consistent relativistic quasiparticle random-phase approximation (RQRPA), based on effective Lagrangians with density-dependent meson-nucleon couplings, and formulated in the relativistic Hartree-Bogoliubov canonical single-particle basis.

\section{ACKNOWLEDGMENTS}

This work has been supported in part by the Bundesministerium für Bildung und Forschung under project 06 TM 979, and by the Gesellschaft für Schwerionenforschung (GSI) Darmstadt. T. N. acknowledges the support from the Alexander von Humboldt Stiftung.

[1] P. Ring, Prog. Part. Nucl. Phys. 37, 193 (1996).

[2] Z.Y. Ma, N. Van Giai, H. Toki, and M. L'Huillier, Phys.Rev. C 42, 2385 (1997).

[3] D. Vretenar, A. Wandelt, and P. Ring, Phys. Lett. B 487, 334 (2000).

[4] J.F. Dawson and R.J. Furnstahl, Phys. Rev. C 42, 2009 (1990).

[5] P. Ring, Zhong-yu Ma, Nguyen Van Giai, D. Vretenar, A. Wandelt, and Li-gang Cao, Nucl. Phys. A694, 249 (2001).

[6] Z.Y. Ma, N. Van Giai, A. Wandelt, D. Vretenar and P. Ring, Nucl. Phys. A686, 173 (2001).

[7] J. Piekarewicz, Phys.Rev. C 64, 024307 (2001).

[8] Z.Y. Ma, A. Wandelt, N. Van Giai, D. Vretenar, P. Ring and L.G. Cao, Nucl. Phys. A703, $222(2002)$.

[9] D. Vretenar, N. Paar, P. Ring and G. A. Lalazissis, Phys. Rev. C 63, 047301 (2001).

[10] D. Vretenar, N. Paar, P. Ring and G. A. Lalazissis, Nucl. Phys. A692, 496 (2001).

[11] D. Vretenar, N. Paar, T. Nikšić, and P. Ring, Phys. Rev. C 65, 021301 (2002).

[12] C. Fuchs, H. Lenske, and H.H. Wolter, Phys. Rev. C 52, 3043 (1995).

[13] S. Typel and H.H. Wolter, Nucl. Phys. A656, 331 (1999).

[14] C.M. Keil, F. Hofmann, and H. Lenske, Phys. Rev. C 61, 064309 (2000).

[15] F. Hofmann, C.M. Keil, and H. Lenske, Phys. Rev. C 64, 025804 (2001). 
[16] F. Hofmann, C.M. Keil, and H. Lenske, Phys. Rev. C 64, 034314 (2001).

[17] T. Nikšić, D. Vretenar, P. Finelli, and P. Ring, Phys. Rev. C 66, 024306 (2002).

[18] W. Pöschl, D. Vretenar, G.A. Lalazissis, and P. Ring, Phys. Rev. Lett. 79, 3841 (1997).

[19] D. Vretenar, H. Berghammer, and P. Ring, Nucl. Phys. A581, 679 (1995).

[20] F. de Jong and H. Lenske, Phys. Rev. C 57, 3099 (1998).

[21] J.P. Blaizot, Phys. Rep. 64, 171 (1980).

[22] J.P. Blaizot, J.F. Berger, J. Dechargé, and M. Girod, Nucl. Phys. A591, 435 (1995).

[23] M. Farine, J.M. Pearson, and F. Tondeur, Nucl. Phys. A615, 135 (1997).

[24] D. Vretenar, G.A. Lalazissis, R. Behnsch, W. Pöschl and P. Ring, Nucl. Phys. A621, 853 (1997).

[25] D.H. Youngblood, H.L. Clark, and Y.W. Lui, Phys. Rev. Lett. 82, 691 (1999).

[26] J. Piekarewicz, nucl-th/0205007.

[27] C.-H. Lee, T.T.S. Kuo, G.Q. Li, and G.E. Brown, Phys. Rev. C 57, 3488 (1998).

[28] R.J. Furnstahl, Nucl. Phys. A706, 85 (2002).

[29] V.E. Starodubsky and N.M. Hintz, Phys. Rev. C 49, 2118 (1994).

[30] A. Krasznahorkay et al., Nucl. Phys. A567, 521 (1994).

[31] P.-G. Reinhard, Nucl. Phys. A649, 305c (1999).

[32] J. Ritman et al., Phys. Rev. Lett. 70, 533 (1993).

[33] C. Mahaux and R. Sartor, Adv. Nucl. Phys. 20, 1 (1996).

[34] M. Jaminon and C. Mahaux, Phys. Rev. C 40, 354 (1989).

[35] M. Jaminon and C. Mahaux, Phys. Rev. C 41, 697 (1990).

[36] F. E. Bertrand, G.R. Satchler, D.J. Horen, and A. van der Woude, Phys. Lett. B 80, 198 (1979).

[37] A. Leistenschneider et al., Phys. Rev. Lett. 86, 5442 (2001).

[38] A. Zilges, S. Volz, M. Babilon, T. Hartmann, P. Mohr and K. Vogt, Phys. Lett. B 542, 43 (2002). 
TABLE I: The effective interaction DD-ME1. See Eqs. (37)- (39) for the definition of the coupling parameters.

\begin{tabular}{cc} 
& DD-ME1 \\
\hline \hline$m_{\sigma}$ & 549.5255 \\
$m_{\omega}$ & 783.0000 \\
$m_{\rho}$ & 763.0000 \\
$g_{\sigma}\left(\rho_{\text {sat }}\right)$ & 10.4434 \\
$g_{\omega}\left(\rho_{\text {sat }}\right)$ & 12.8939 \\
$g_{\rho}\left(\rho_{\text {sat }}\right)$ & 3.8053 \\
$a_{\sigma}$ & 1.3854 \\
$b_{\sigma}$ & 0.9781 \\
$c_{\sigma}$ & 1.5342 \\
$d_{\sigma}$ & 0.4661 \\
$a_{\omega}$ & 1.3879 \\
$b_{\omega}$ & 0.8525 \\
$c_{\omega}$ & 1.3566 \\
$d_{\omega}$ & 0.4957 \\
$a_{\rho}$ & 0.5008
\end{tabular}

FIG. 1: Density-dependent RRPA peak energies of the ISGMR in ${ }^{208} \mathrm{~Pb}$ as a function of the nuclear matter compressibility $K_{\infty}$. The calculated peaks are shown in comparison with the experimental excitation energy of the monopole resonance: $E=14.1 \pm 0.3 \mathrm{MeV}$ [25].

FIG. 2: The isoscalar monopole strength distribution (left panel) and transition densities (right panel) in ${ }^{208} \mathrm{~Pb}$, calculated with a density-dependent effective interaction with $K_{\infty}=270 \mathrm{MeV}$. The proton, neutron and total isoscalar transition densities correspond to the peak at $E=14.1$ $\mathrm{MeV}$. 
FIG. 3: The IVGDR excitation energy of ${ }^{208} \mathrm{~Pb}$ (upper left panel), and the parameter $p_{0}$ of the linear density dependence of the nuclear matter asymmetry energy, as functions of the volume asymmetry $a_{4}$. The shaded area denotes the experimental IVGD resonance energy $13.3 \pm 0.1 \mathrm{MeV}$. In the right panel the asymmetry energy curves, as functions of the baryon density, are plotted for different values of the volume asymmetry $a_{4}$.

FIG. 4: The isovector dipole strength distribution (left panel) and transition densities (right panel) in ${ }^{208} \mathrm{~Pb}$, calculated with a density-dependent effective interaction with $a_{4}=35 \mathrm{MeV}$. The proton, neutron and total isovector transition densities correspond to the peak at $E=13.3 \mathrm{MeV}$.

FIG. 5: The IVGDR excitation energy of ${ }^{208} \mathrm{~Pb}$ (upper left panel), and the parameter $p_{0}$ of the linear density dependence of the nuclear matter asymmetry energy, as functions of the volume asymmetry $a_{4}$. The shaded area denotes the experimental IVGD resonance energy $13.3 \pm 0.1 \mathrm{MeV}$. In the right panel the asymmetry energy curves, as functions of the baryon density, are plotted for different values of the volume asymmetry $a_{4}$.

FIG. 6: Average energy gap between the last occupied and first unoccupied major shells in ${ }^{208} \mathrm{~Pb}$, as function of the isoscalar parameter $\delta(46)$. The average gaps of neutron states are denoted by dots, and those of proton states by squares.

FIG. 7: Centroid energies of the isoscalar quadrupole Hartree response (upper panel), and the ISGQR peak energies calculated in RRPA (lower panel), for five different density-dependent interactions characterized by the parameter $\delta$ (46). As in the previous examples, the calculation is performed for ${ }^{208} \mathrm{~Pb}$. The shaded area denotes the empirical ISGQR excitation energy in ${ }^{208} \mathrm{~Pb}$ : $10.9 \pm 0.3 \mathrm{MeV}$ [36].

FIG. 8: The isoscalar quadrupole strength distribution (left panel) and transition densities (right panel) in ${ }^{208} \mathrm{~Pb}$, calculated for $\delta=0.93$ (see text for description). The vertical bar denotes the $0 \hbar \omega 2^{+}$discrete state. The proton, neutron, and total isoscalar transition densities correspond to the ISGQR peak at $E=11.2 \mathrm{MeV}$ excitation energy. 